\title{
АНАЛИЗ ЭНЕРГЕТИЧЕСКИХ ПОКАЗАТЕЛЕЙ ТЯГОВОГО ВЫПРЯМИТЕЛЯ С ШИРОТНО-ИМПУЛЬСНЫМ РЕГУЛИРОВАНИЕМ
}

Д-р техн. наук В. Г. Ягуп, асп. Е.Я. Ивакина, канд. техн. наук Е.В. Ягуп

\section{АНАЛІЗ ЕНЕРГЕТИЧНИХ ПОКАЗНИКІВ ТЯГОВОГО ВИПРЯМЛЯЧА 3 ШИРОТНО-ІМПУЛЬСНОЮ МОДУЛЯЦІЕЮ}

Д-р техн. наук В. Г. Ягуп, асп. К.Я. Івакина, канд. техн. наук К.В. Ягуп

\section{ANALYSIS OF POWER FACTOR OF TRACTION RECTIFIER WITH PULSE-WIDTH CONTROL}

Yagup V.G., Ivakina E.Y., Yagup E.V.

В статье выполнен анализ энергетических показателей выпрямительной установки тяговой подстанциии с вольтодобавочным преобразователем, управляемым ишротно-импульсной модуляцией. Получено аналитическое выражение для коэффиџиента мощзности с учетом влияния на него коэффициента трансформации и коэффициента заполнения импульсов ШИМ.

Ключевые слова: выпрямитель управляемый, ииротно- импульсная модуляция, коэффициент мощцности.

У статті виконано аналіз енергетичних показників випрямляючої установки тягової підстаниії з вольтододатним перетворювачем, керованим ииротно-імпульсною модуляцією. Одержано аналітичний вираз для коефіцієнта потужності з урахуванням впливу на нього коефічієнта трансформаџіï та коефіцієнта заповнювання імпульсів ШИМ.

Ключові слова: випрямляч керований, широтно- імпульсна модуляція, коефіuієнт потужності.

In the article a rectifier for the feed of electric transport is considered. This rectifier consists of the serial united bridge circuit of rectifying, which are connected in series. The first rectifier creates basic part of output voltage, and the second is used for control and stabilization of supply voltage. It is suggested to carry out control by pulse-width control method. A basic power index-power-factor is analysed. For its finding an original method which is based on equality of active-powers, which consumed from a network and given in loading is offered. It allows to exclude bulky procedure of harmonic analysis and get analytical character expression for a power-factor. Such parameters, as coefficient of transformation and duration of pulse-width control, are taken into account. By MATHCAD was built family of plots which shows, that the offered system possesses high power indexes, that promotes substantially economies of electric power.

Keywords: control rectifier, pulse-width modulation, power factor.

Введение. Проблемы создания высокоэффективных систем электроснабжения решаются в последние годы с использованием современных средств энергетической электроники [1].

Постановка проблемы. Для питания тяговых нагрузок в электротранспорте рациональным оказывается использование выпрямителей с вольтодобавкой [2]. При этом основной выпрямитель, обеспечивающий большую часть питающего напряжения, питается от трансформатора, выполненного по схеме "звезда-звезда". Добавочный выпрямитель обеспечивает до 20-30\% 
напряжения тяговой подстанции, собирается по схеме питания "звезда-треугольник". На этот выпрямитель возлагаются функции регулирования и стабилизации выходного напряжения тяговой подстанции, и потому он выполняется на управляемых силовых полупроводниковых приборах. Применение такого принципа повышает энергетические показатели системы электроснабжения даже в случае регулирования выпрямителя изменением угла управления тиристоров.

Анализ состояния вопроса. Разработка быстродействующих силовых полупроводниковых приборов $[3,4]$ дала возможность реализовать самые различные методы управления потоками электрической энергии. В частности, эффективным оказывается применение широтно-импульсной модуляции для регулирования и стабилизации напряжения.

Определение цели и задачи исследований. Целью исследования является анализ энергетических показателей тягового выпрямителя с добавочным выпрямителем, регулирование напряжения которого осуществляется с помощью ШИМ.

Основная часть исследований. Структурная схема выпрямителя с вольтодобавкой, регулируемой по принципу ШИМ, приведена на рис. 1.

Здесь тяговый двигатель ТД питается от последовательно включенных неуправляемого НВ и управляемого выпрямителей УВ с модулятором ШИМ. Будем считать ток в ТД идеально сглаженным с постоянным значением $\mathrm{I}_{0}$. Силовые полупроводниковые приборы преобразовательных устройств принимаем идеальными. Питающие трансформаторы Тр1 (по схеме "звезда-звезда") и ТР2 (по схеме "звезда-треугольник") также считаем идеальными. Их коэффициенты трансформации, определяемые отношением витков вторичной обмотки к количеству витков первичной обмотки, обозначим $k_{1}$ и $k_{2}=k$.

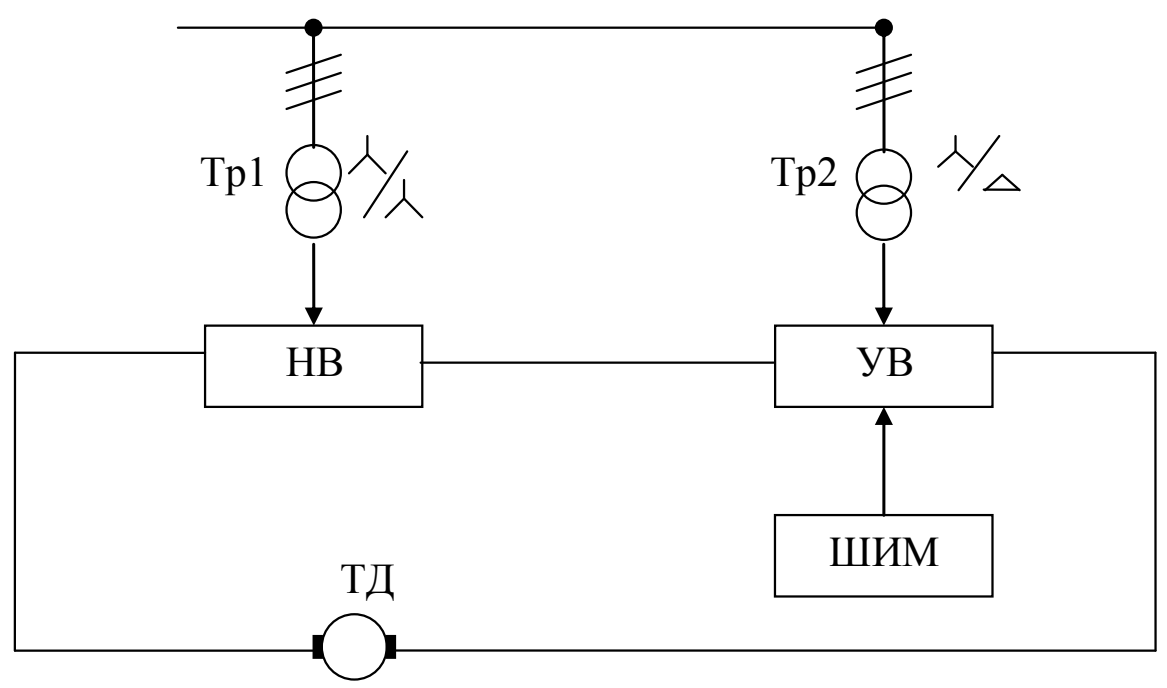

Рис. 1. Схема тягового выпрямителя

Питающую сеть будем полагать бесконечно мощной и представленной в виде источников напряжений $\mathrm{e}_{\mathrm{a}}(\mathrm{t}), \mathrm{e}_{\mathrm{b}}(\mathrm{t}), \mathrm{e}_{\mathrm{c}}(\mathrm{t})$. Период питающих напряжений равен $2 \pi$.

Учитывая условия идеализации, можно изобразить упрощенную схему преобразователя в следующем виде (рис. 2).

Ток в каждой фазе питающей сети формируется благодаря трансформации в обратном направлении постоянного тока $\mathrm{I}_{0}$ через блок коммутаторов и блок трансформаторов. Сетевой ток определяется суммой составляющих первичных обмоток (рис. 3).

$$
i_{c}=i_{Y}+i_{\Delta}
$$

где $i_{c}-$ сетевой ток одной из фаз; 
$i_{Y}$ - ток первичной обмотки трансформатора, включенного по схеме "звезда-звезда";
$i_{\Delta}-$ ток первичной обмотки трансформатора, включенного по схеме "звездатреугольник".

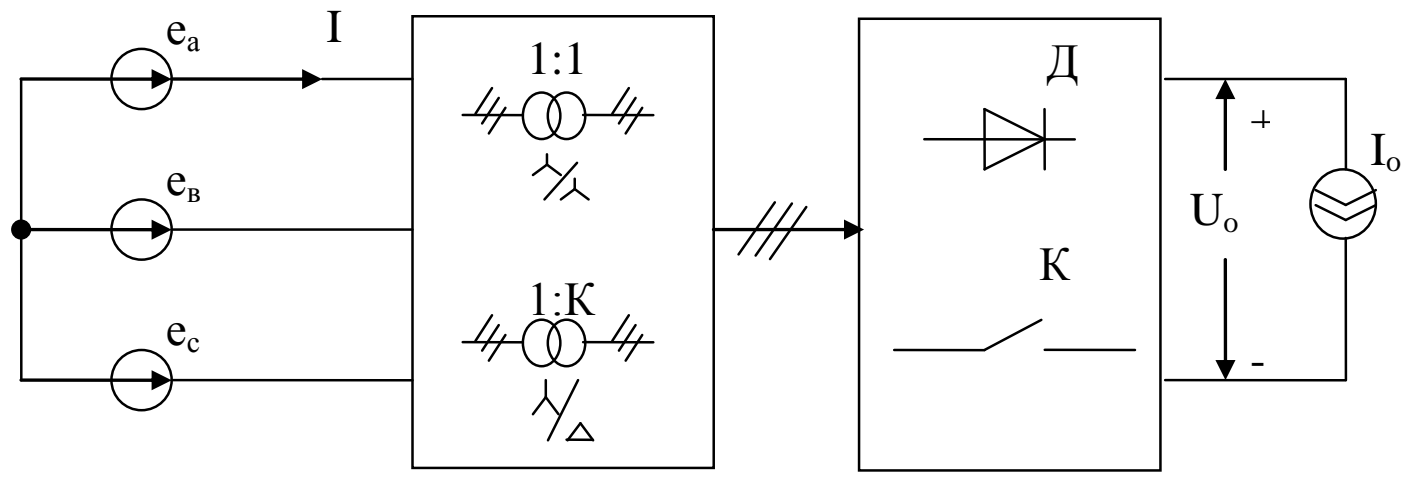

БT
БК

Рис. 2. Идеализированная модель преобразователя

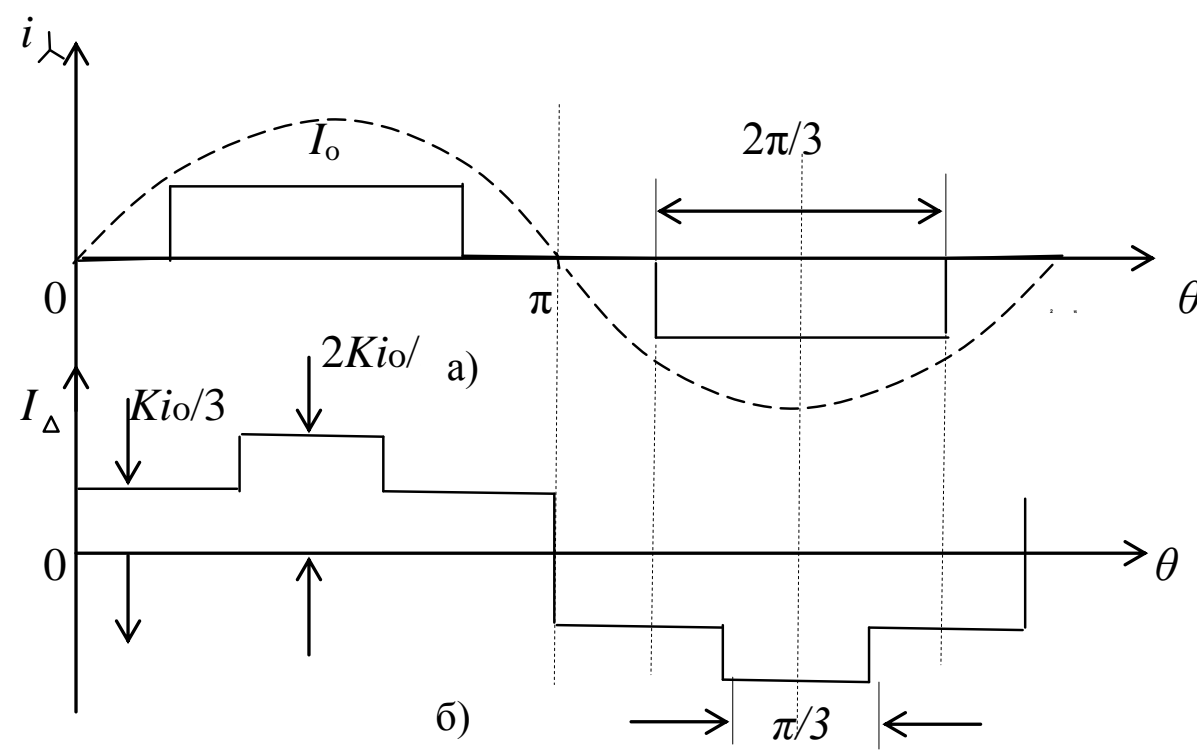

Рис. 3. Составляющие сетевого тока без ШИМ

Временные диаграммы показывают характер изменений первичных токов основного (рис. 3, a) и вольтодобавочного (рис. 3, б) выпрямителей без учета ШИМ. Значения первичных токов вольтодобавочного выпрямителя $\frac{k I_{0}}{3}$ и $\frac{2 k I_{0}}{3}$ учитывают включение вторичной обмотки по схеме треугольника, а также коэффициент трансформации $k=k_{2}<1$. Напряжение фазы А имеет нулевой сдвиг (рис. 3 ,a, пунктирная линия).

Интегральным

показателем, характеризующим энергетические свойства вентильного преобразователя, является коэффициент мощности [5], который при абсолютно жесткой сети определяется соотношением 


$$
K_{\mathcal{M}}=K_{{ }} K_{c}
$$

где $K_{И}-$ коэффициент искажения кривой сетевого тока;

$$
K_{C}-\text { коэффициент сдвига. }
$$

Коэффициент искажения $K_{И}$ определяется отношением действующего значения первой гармоники $I_{1}$ сетевого тока к его действующему значению $I_{\partial}$ :

$$
K_{M}=\frac{I_{1}}{I_{\partial}}
$$

Коэффициент сдвига представляет собою косинус угла сдвига $\varphi$ первой гармоники сетевого тока относительно напряжения соответствующей фазы питающей сети

$$
K_{c}=\cos \varphi
$$

Таким образом,

$$
K_{M}=\frac{I_{1} \cos \varphi}{I_{\partial}}
$$

Вычисление действующего значения в соответствии с его определением как среднеквадратичного за период $\mathrm{T}$ значения должно осуществляться по соответствующей формуле [6]:

$$
I_{\partial}=\sqrt{\frac{1}{T} \int_{0}^{T}[i(t)]^{2} d t}
$$

Определение $I_{1}$ и $\cos \varphi$ предполагает в общем случае проведение гармонического анализа, выявляющего косинусную $I_{1 c}$ и синусную $I_{1 s}$ составляющие первой гармоники, после чего угол сдвига определяется из выражения

$$
\operatorname{tg} \varphi=\frac{I_{1 s}}{I_{1 c}}
$$

Рассматривая идеализированную модель преобразователя, учтем, что блоки БТ и БК не потребляют активной мощности. Тогда активная мощность на выходе преобразователя $P_{0}$ и активная мощность $P_{A}$, потребляемая от сети, должны быть равны. Активная мощность нагрузки определяется соотношением

$$
P_{0}=I_{0} U_{0},
$$

где $U_{0}$ - среднее значение выпрямленного напряжения.

Активная мощность фазы А определится из выражения

$$
P_{a}=I_{1} \frac{E_{m}}{\sqrt{2}} \cos \varphi
$$

где $E_{m}-$ амплитудное значение фазного напряжения питающей сети.

Активная мощность $P_{A}$, потребляемая всей преобразовательной системой, обеспечивается всеми тремя фазами сети:

$$
P_{A}=3 P_{a}=\frac{3}{\sqrt{2}} E_{m} \cdot I_{1} \cos \varphi .
$$

Следовательно, может быть записано уравнение

$$
P_{0}=P_{A}
$$

которое при соответствующих подстановках приобретет вид

$$
I_{0} U_{0}=\frac{3}{\sqrt{2}} E_{m} \cdot I_{1} \cos \varphi .
$$

Из последнего выражения определяется числитель выражения (5) для искомого коэффициента мощности

$$
I_{1} \cos \varphi=\frac{\sqrt{2} I_{0} U_{0}}{3 I_{\partial} E_{m}} .
$$

Таким образом, вычисляя правую часть выражения (13), можно косвенным образом вычислить те величины, которые при формальном подходе потребовали бы гармонического анализа. Действуя предложенным образом, можно не вычислять 
активную и реактивную мощности в сети для определения угла сдвига, как это, например, предлагается в [7]. Окончательно получим

$$
K_{M}=\frac{\sqrt{2} I_{0} U_{0}}{3 I_{\partial} E_{m}} .
$$

Для вычисления среднего значения $U_{d}$ выпрямленного напряжения многотактного выпрямителя воспользуемся известным выражением [8].

$$
U_{d}=\frac{m}{\pi} E_{m} \sin \frac{\pi}{m}
$$

где $m-$ число тактов выпрямленного напряжения;

$$
E_{m} \quad-\text { амплитуда эквивалентного }
$$

синусоидального напряжения на питающей выпрямитель обмотке, которое передается на выход выпрямителя.

Для неуправляемого выпрямителя составляющая $K_{Y}$ полного выходного напряжения с учетом $K_{1}=1$

$$
U_{Y}=\frac{m}{\pi} \sqrt{3} E_{m} \sin \frac{\pi}{m} .
$$

Для управляемого выпрямителя нужно учитывать включение обмоток по схеме"звездатреугольник", коэффициент трансформации $k_{2}=k$ и коэффициент заполнения $\gamma$ импульсом периода широтно-импульсной модуляции. Тогда составляющая $U_{\Delta}$ выпрямленного напряжения управляемого выпрямителя определится выражением

$$
U_{\Delta}=\gamma k \frac{m}{\pi} E_{m} \sin \frac{\pi}{m}
$$

Среднее значение полного напряжения исследуемого выпрямителя

$$
U_{0}=U_{Y}+U_{\Delta}=\frac{m}{\pi} E_{m} \sin \frac{\pi}{m}(\sqrt{3}+\not k) .
$$

Обратим внимание на то обстоятельство, что в периоде сетевого тока содержится четыре этапа, которые дадут одинаковые значения интеграла на интервале в четверть периода (рис. 3). Поэтому можно ограничиться рассмотрением лишь первого из этих этапов (рис. 4).

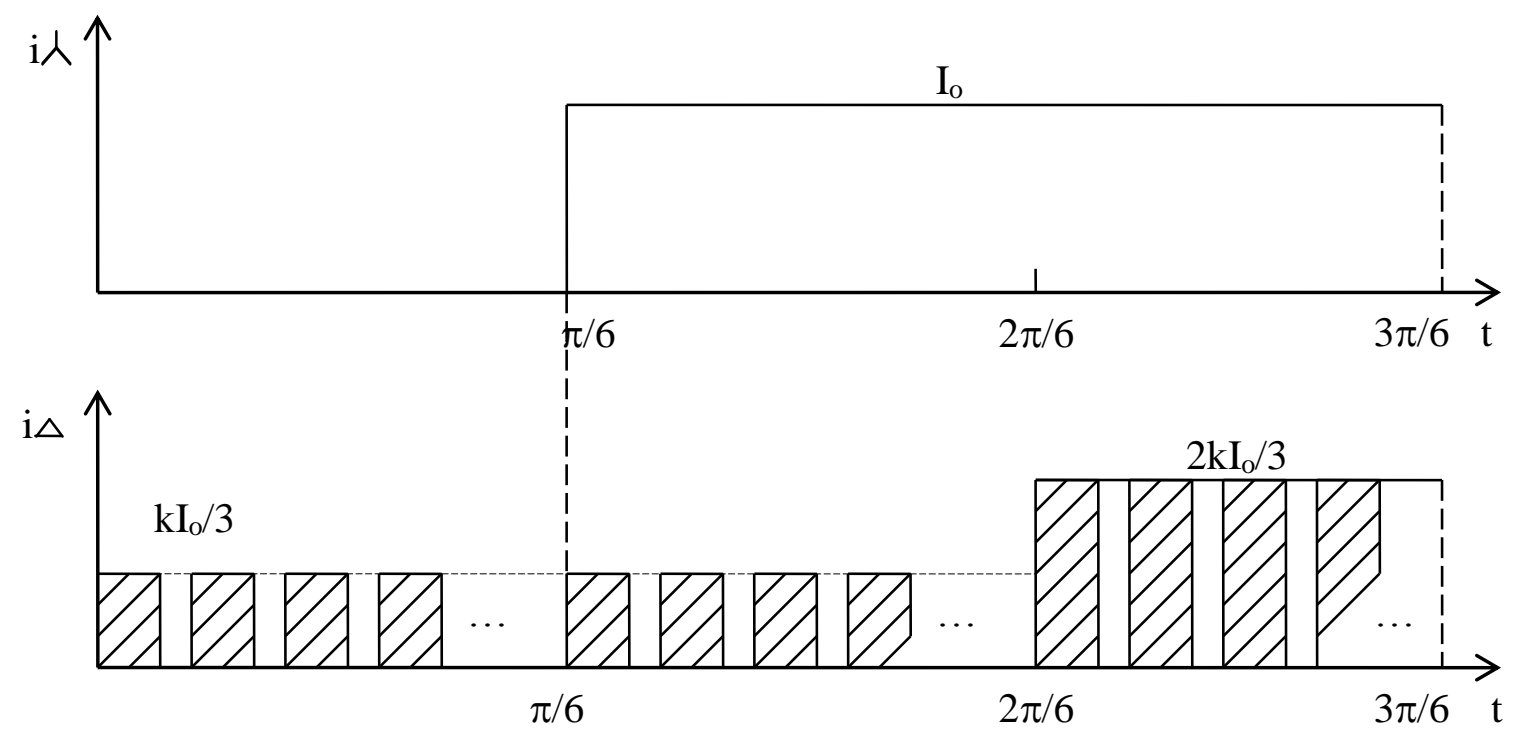

Рис. 4. Составляющие сетевого тока с учетом ШИМ 
На этом этапе содержится три интервала, длительность каждого составляет $\frac{\pi}{6}$. Рассмотрим взятие интеграла от квадратичного значения в случае, когда одна составляющая постоянна и имеет амплитуду $I_{1 m}$, а вторая составляющая имеет амплитуду $I_{2 m}$ и подвергается воздействию ШИМ с коэффициентом заполнения $\gamma$ и целым количеством $\mathrm{n}$ импульсов на интервале $\frac{\pi}{6}$. В этом случае длительность периода ШИМ

$$
t_{u}=\frac{\pi}{6 n}
$$

Длительность импульса ШИМ составляет величину $\frac{\gamma \pi}{6 n}$, а длительность паузы ШИМ занимает остальную часть периода $t_{u}$ и равна $\frac{(1-\gamma) \pi}{6 n}$

Тогда в общем случае интеграл от квадратичного значения определится суммой соответствующих прямоугольных площадок .

$$
\int_{t_{0}}^{t_{0}+\frac{\pi}{6}} i^{2} d t=n\left[\left(I_{1 m}+I_{2 m}\right)^{2} \frac{\gamma \pi}{6 n}+I_{1 m}^{2} \frac{(1-\gamma) \pi}{6 n}\right]
$$

После упрощения первой части придем к окончательному выражению

$$
\int_{t_{0}}^{t_{0}+\frac{\pi}{6}} i^{2} d t=\frac{\pi}{6}\left[I_{1 m}^{2}+\gamma I_{2 m}^{2}+2 \gamma I_{1 m} I_{2 m}\right]
$$

Дальнейший расчет сводится к учету амплитуд составляющих сетевого тока для каждого интервала рассматриваемого первого этапа.

$$
\text { Для интервала } t_{0}=0: I_{1 m}=0, I_{2 m}=\frac{k I_{0}}{3} \text { : }
$$

$$
\left.\int_{t_{0}}^{\frac{\pi}{6}} i^{2} d t=\frac{\pi}{6} \gamma \frac{k^{2} I_{0}^{2}}{9}\right]
$$

Для интервала $t_{0}=\frac{\pi}{6}: \quad I_{1 m}=I_{0}$, $I_{2 m}=\frac{k I_{0}}{3}$ :

$$
\int_{\pi}^{\frac{2 \pi}{6}} i^{2} d t=\frac{\pi}{6}\left(I_{0}^{2}+\gamma \frac{k^{2} I_{0}^{2}}{9}+2 \gamma I_{0} \frac{k I_{0}}{3}\right]
$$

Для интервала $t_{0}=\frac{2 \pi}{6}: \quad I_{1 m}=I_{0}$, $I_{2 m}=\frac{2 k I_{0}}{3}$ :

$$
\int_{\frac{2 \pi}{6}}^{\frac{3 \pi}{6}} i^{2} d t=\frac{\pi}{6}\left(I_{0}^{2}+\gamma \frac{4 k^{2} I_{0}^{2}}{9}+2 \gamma I_{0} \frac{k I_{0}}{3}\right]
$$

Суммируя значения интегралов по каждому интервалу, получим

$$
\int_{0}^{\frac{\pi}{2}} i^{2} d t=\frac{\pi}{6} I_{0}^{2} \cdot \frac{2}{3}\left(1+\frac{\gamma k^{2}}{3}+\gamma_{k}\right)
$$

На интервале периода от 0 до $2 \pi$ значение интеграла следует учетверить, и после извлечения корня получим следующее выражение для действующего значения сетевого тока одной фазы:

$$
I_{\partial}=I_{0} \sqrt{\frac{2}{3}\left(1+\frac{\gamma k^{2}}{3}+\gamma k\right)}
$$

Подставляя выражения для $U_{0}$ по формуле (18) и выражение для $I_{\partial}$ по формуле (26) в формулу (14) для вычисления коэффициента мощности, получим

$$
K_{M}=\frac{\sqrt{2} \cdot I_{0} \frac{m}{\pi} E_{m} \sin \frac{\pi}{m}(\sqrt{3}+\gamma k)}{3 \cdot I_{0} \sqrt{\frac{2}{3}\left(1+\frac{\gamma k^{2}}{3}+\gamma k\right)} E_{m}} .
$$




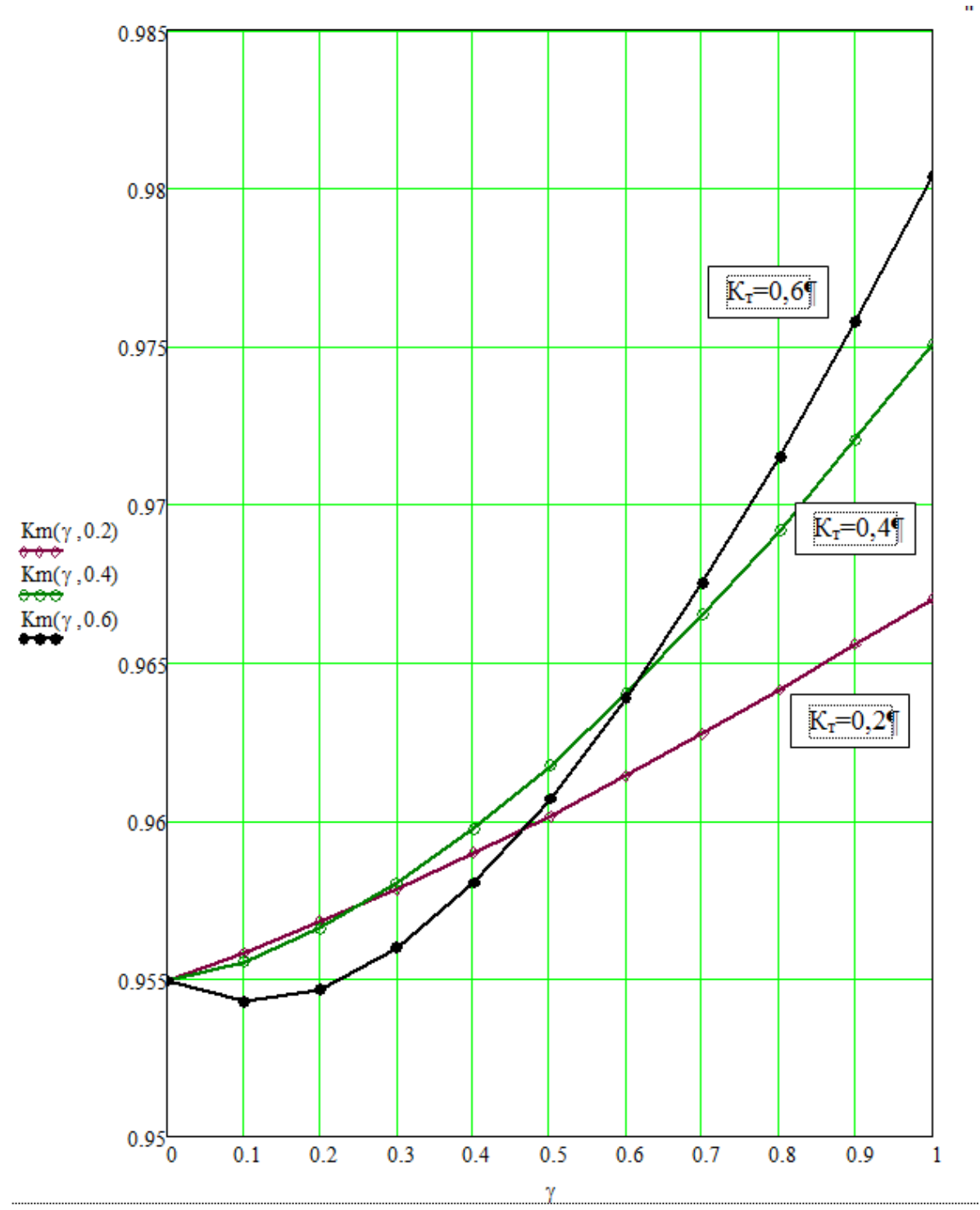

Рис.5. Зависимости коэффициента мощности выпрямителя с вольтодобавкой и ШИМ от $\mathrm{K}_{\mathrm{T}}$ и $\gamma$

C помощью программы MathCAD построены графики (см. рис. 5), представляющие семейство зависимостей коэффициента мощностей при вариации коэффициента заполнения от 0 до 1 при значениях коэффициента трансформации 0,2 ; 0,$4 ; 0,6$. Анализ этих графиков позволяет оценить энергетические показатели и сделать выводы по выбору указанных параметров.

Выводы. На основе баланса активных мощностей на входе и выходе выпрямителя обоснован способ вычисления коэффициента мощности, позволяющий вывести символьное выражение без проведения громоздкого гармонического анализа. Получено аналитическое выражение для коэффициента мощности исследуемого выпрямителя с учетом коэффициента заполнения ШИМ и коэффициента трансформации для вольтодобавочного выпрямителя. Построено семейство графиков для коэффициента мощности, анализ которого позволяет рекомендовать значение коэффициента трансформации для вольтодобавочного выпрямителя в диапазоне $0,2 \div 0,4$, когда обеспечивается практически линейная зависимость коэффициента мощности от коэффициента заполнения импульсов ШИМ. 


\section{Список использованных источников}

1. Acha E. Power Electronic Control in Electric/ Acha E., Anaya - Lara O., Agelidis V. G. // Newness Power Eng., 1st ed. New York: Oxford, 2002. - 277 p.

2. Бей, Ю.М. Тяговые подстанции [Текст] / Ю.М. Бей, Р.Р, Мамошин, В.Н. Пупырин, М.Г. Шалимов. - М.: Транспорт, 1986. - 319 с.

3. Power electronics technology: Present trends and future developments// Pros. IEEE. 2001. Vol.89. №6.

4. Matsuda H. New Advanced Power Semiconductors Toshiba Corporation. Power Conversion. May 1999. - p. 139.

5. Csaki F. Power Electronics: Problems Manual/ Csaki F., Ipsits I., Karpati A., Magyar P. Budapest, 1979. -382.

6. Arrillaga J. Power system harmonics/ Arrillaga J., Watson N. - Wiley. 1, 2003. - 412 p.

7. Маевский, О.А. Энергетические показатели вентильных преобразователей [Текст] / О.А. Маевский.. - М.: Энергия, 1978. - 320 с.

8. Дьяконов, B. MathCAD 2000 [Текст] / В. Дьяконов. - С.Пб.: Питер, 2001. - 592 с.

Ягуп Валерій Григорович, доктор техн. наук, професор кафедри автоматизованих систем електричної тяги Української державної академії залізничного транспорту. Тел. (057)730-10-76.

Ягуп Катерина Валеріївна, канд. техн. наук, доцент кафедри автоматизованих систем електричної тяги Украінської державної академії залізничного транспорту. Тел. (057)730-10-76.

Івакіна Катерина Яківна, аспірант Харківського національного університету міського господарства ім.О.М.Бекетова, кафедра електропостачання міст.

Yagupov Valeriy Grigorovich, Dr. tehnichny Sciences, Professor Departments avtomatizovani sistemi elektrichno End Ukraïnskoï derzhavnoï akademi Zaliznicnovo transport. Tel. (057) 730-10-76.

Yagupov Katerina Valeriïvna, candidate tehnichny Sciences. Associate Professor, Department avtomatizovani sistemi elektrichno End Ukrainskoï derzhavnoï akademiï Zaliznicnovo transport. Tel. (057) 730-10-76.

Ivakina Katerina Yakivna postgraduate Kharkiv National University of Municipal Management named after A.N. Beketova Dept. of electric power supply of the cities. 